\title{
Nonthermal Distribution of Electro-negative Light-ions in Heavy-ion-acoustic Solitary and Shock Structures
}

\author{
M. M. Haider ${ }^{1}$, I. Sultana ${ }^{1}$, S. Khatun ${ }^{1}$, J. Nasrin ${ }^{1}$, N. Tasnim ${ }^{1}$, O. Rahman ${ }^{1}$ and S. Akter ${ }^{2 *}$ \\ ${ }^{1}$ Department of Physics, Mawlana Bhashani Science and Technology University, Santosh, Tangail-1902, \\ Bangladesh \\ ${ }^{2}$ Department of Physics, Jahangirnagar University, Savar, Dhaka-1342, Bangladesh \\ Email: liza.phy@gamil.com
}

\begin{abstract}
An attempt has been taken to study the nonlinear propagation of electro-positive heavy ion-acoustic (EPHIA) solitary and shock waves in multi-component plasmas containing mobile electro-positive heavy ions (EPHIs), nonthermal distributed electro-negative light ions (ENLIs) and Maxwellian electrons. The effects of temperature, mobile EPHIs, ENLIs and its nonthermality on solitary and shock waves are investigated in present work. To do so, we have derived the standard Korteweg-de Vries (K-dV) and Burger equations and their solutions. We have studied the effects of the parametric regimes, which affect the solitary and shock waves, numerically as well as graphically.
\end{abstract}

Keywords: Electro-positive heavy ion, solitary and shock waves, nonthermal distribution.

\section{Introduction}

Ion-acoustic (IA) solitary waves (SWs) are one of the important non-linear phenomena in plasma systems. Ions may be present in most of the plasmas and the presence of ions leads to existence of several lowfrequency waves. IA SWs in unmagnetized plasma have been studied by a number of authors both experimentally and theoretically. Ions are considered as electro-positive, in most of the earlier works, but Conney et al. [1] found the existence of electro-positive (EP) and electro-negative (EN) ions plasma in their experimental investigation. The latter may also support the formation of IA shocks when the ratio of the negative ion to positive ion number density exceeds about 0.9 [2]. The EP and EN ions in plasmas are found in natural and technological environments like the D-region of the Earth's ionosphere, the Earth's mesosphere, the solar photosphere, and the microelectronics plasma processing reactors [3]. In the recent few years, the study of Korteweg-de Vries (K-dV) and Burger ion-acoustic solitons and shocks in a multi-species plasma consisting of electro-positive ions (EPIs), electro-negative ions (ENIs), and electrons become the field of current research investigation. Mishra et al. [4] studied the obliquely propagating ion-acoustic solitary waves in a multi-component magnetized plasma consisting of warm adiabatic positive and negative ion species and hot isothermal electrons. Haider et al. have studied the nonlinear propagation of IA solitary waves with EPIs and ENIs for Maxwellian [6,5], trapped [7,8], and nonthermal $[9,10]$ distributed electrons. Nakamura and Tsukabayashi [11] have studied experimentally the propagation of ion-acoustic solitons in a plasma with negative ions.

The effects of heavy ions on radio wave propagation in the ionosphere have been studied by Hines [12] considering a multi-ion plasma around sixty years ago. Recently Hossen et al. [13] showed that heavy ions significantly modify the basic features of heavy-ion-acoustic (HIA) waves in degenerate plasmas. Ema et al. [14] have studied the dust-ion-acoustic (DIA) shock structures in multi-ion unmagnetized plasmas containing negatively charged heavy ions, positively charged light ions following Maxwellian distribution, and nonextensive electrons. Shah et al. [15] have theoretically investigated properties of HIA solitary and shock waves in unmagnetized plasmas with Maxwellian light ions. All of the above investigations, light ions are considered as Maxiwellian. Very recently Rahaman and Haider [16] considered that ENLIs as trapped. But the ENLIs might very cold and following the nonthermal distribution. Therefore a necessity is felt to study the effect of nonthermal ENLIs in electro-positive HIA (EPHIA) solitary and shock waves in multi-ion plasmas. In the present work, the propagation of EPHIA solitary structures have been studied in unmagnetized plasma consisting of EPHIs, ENLIs following nonthermal distribution and Maxwellian 
distributed electrons. The reductive perturbation method [17] has been employed to derive the K-dV [18] and Burgers [19] equations.

The rest of the manuscript is organized as follows. The plasma model for a small but finite amplitude EPHIA solitary and shock waves in an unmagnetized collisionless multi-component plasma with nonthermal ENLIs is presented in section 2. The well known K-dV equation is derived with its solution to study the solitary waves in section 3. To study the shock waves, Burgers equation is derived as well as its solution in section 4 . The numerical study of this work is presented in section 5 . Finally, a summary of our results is discussed in section 6 .

\section{Plasma Model}

We have considered a collisionless, unmagnetized multi-ion plasma system where -

i) EPHIs are mobile,

ii) ENLIs are nonthermal,

iii) Electrons follow the Maxwellian distribution.

The one dimensional dynamics (equation of continuity, momentum equation and Poisson's equation) of the EPHIA waves in the normalized form are governed by

$$
\begin{aligned}
& \frac{\partial n_{p}}{\partial t}+\frac{\partial}{\partial x}\left(n_{p} u_{p}\right)=0 \\
& \frac{\partial u_{p}}{\partial t}+u_{p} \frac{\partial u_{p}}{\partial x}=-\frac{\partial \phi}{\partial x}+\eta \frac{\partial^{2} u_{p}}{\partial x^{2}} \\
& \frac{\partial^{2} \phi}{\partial x^{2}}=\mu_{e} n_{e}+\mu_{n} n_{n}-n_{p}
\end{aligned}
$$

where, $n_{p}\left(n_{n}\right)$ is the EPHIs (ENLIs) number density normalized by its equilibrium value $n_{p 0}\left(n_{n 0}\right), u_{p}$ is the positive ion fluid speed normalized by $C_{p}=\left(K_{B} T_{e} / m_{p}\right)^{\frac{1}{2}}$ with $K_{B}$ is the Boltzmann constant, $T_{e}$ is the temperature of electrons and $m_{p}$ is the rest mass of EPHIs. $\phi$ is the EPHIA wave potential normalized by $K_{B} T_{e} / e$ with $e$ being the magnitude of the charge of the electron. The time variable (t) is normalized by $\omega_{p}^{-1}=\left(4 \pi n_{p 0} e^{2} / m_{p}\right)^{1 / 2}$ with $c$ being the speed of light. The space variables are normalized by Debye radius $\left.\lambda_{D}=\left(K_{B} T_{e}\right) / 4 \pi n_{p 0} e^{2}\right)$. We have considered the viscous term, i.e. coefficient of viscosity $(\eta)$ which equals to zero at the time of studying solitary waves.

Maxwillan electron distribution can be express as

$$
n_{e}=e^{\phi}
$$

According to Cairns et al. [20] the nonthermal distribution of ENLIs with a population of fast particles $(\gamma)$ can be represented as

$$
n_{n}=\left[1-\sigma_{p} \alpha \phi+\alpha\left(\sigma_{p} \phi\right)^{2}\right] e^{\left(\sigma_{p} \phi\right)}
$$

where $\alpha=4 \gamma /(1+3 \gamma)$ with $\gamma$ represents a parameter determining the fast particles present in this plasma model and $\sigma_{p}\left(=\frac{T_{e}}{T_{n}}\right)$ is the temperature ratio of electron $\left(T_{e}\right)$ to $\operatorname{ENLIs}\left(T_{n}\right)$.

Now using equilibrium charge neutrality condition $n_{e 0}+n_{n 0}=n_{p 0}$, one can write $\mu_{e}=1-\mu_{n}$ where, $\mu_{e}=\frac{n_{e 0}}{n_{p 0}}$ and $\mu_{n}=\frac{n_{n 0}}{n_{p 0}}$.

\section{Solitary Wave}

To derive the Korteweg-de Vries (K-dV) equation from the normalized equations (1)-(3) by employing the reductive perturbation technique, we have introduced stretched co-ordinates as $[7,21]$,

$$
\xi=\epsilon^{\frac{1}{2}}\left(x-v_{p} t\right)
$$




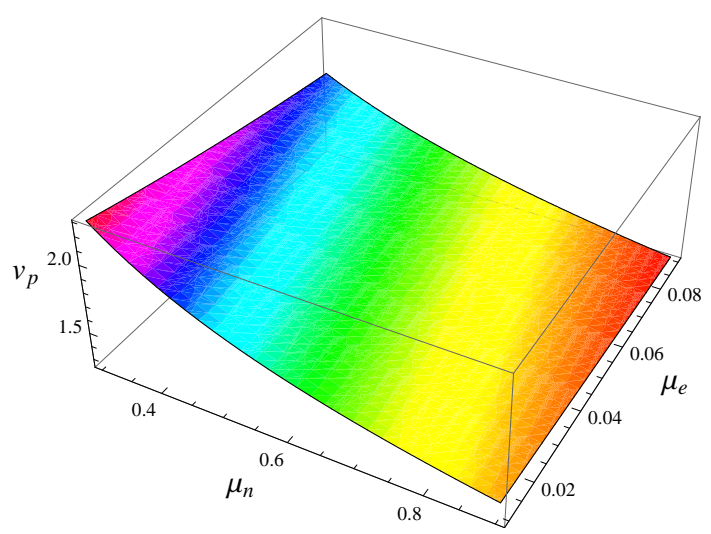

Figure 1. Variation of the phase speed with $\mu_{n}$ and $\mu_{e}$ for $u_{0}=0.1, \alpha=0.7$, and $\sigma_{p}=2$.

$$
\tau=\epsilon^{\frac{3}{2}} t
$$

where $\epsilon$ is the smallness parameter measuring the weakness of the amplitude or dispersion and $v_{p}$ is the phase speed of the system normalized by $C_{p}$.

The perturbed quantities can be expanded about their equilibrium values in powers of $\epsilon$ as

$$
\left.\begin{array}{l}
n_{p}=1+\epsilon n_{p}^{(1)}+\epsilon^{2} n_{p}^{(2)}+\ldots \\
u_{p}=0+\epsilon u_{p}^{(1)}+\epsilon^{2} u_{p}^{(2)}+\ldots \\
\phi=0+\epsilon \phi^{(1)}+\epsilon^{2} \phi^{(2)}+\ldots
\end{array}\right\}
$$

Using the stretched coordinates and Eq.(4) in Eqs. (1)-(3), one can obtain the following linear dispersion relation by taking the lowest order of Eqs. (1)-(3).

$$
v_{p}=\frac{1}{\sqrt{\mu_{e}+\mu_{n} \sigma_{p}(1-\alpha)}}
$$

To get next higher order equations, we have substituted the required parameter values from the above equations into Eqs.(1)-(3).

$$
\begin{aligned}
& \frac{\partial n_{p}^{(1)}}{\partial \tau}-v_{p} \frac{\partial n_{p}^{(2)}}{\partial \xi}+\frac{\partial}{\partial \xi}\left(n_{p}^{(1)} u_{p}^{(1)}\right)+\frac{\partial u_{p}^{(2)}}{\partial \xi}=0 \\
& \frac{\partial u_{p}^{(1)}}{\partial \tau}-v_{p} \frac{\partial u_{p}^{(2)}}{\partial \xi}+u_{p}^{(1)} \frac{\partial u_{p}^{(1)}}{\partial \xi}=-\frac{\partial \phi^{(2)}}{\partial \xi} \\
& \frac{\partial^{2} \phi^{(1)}}{\partial \xi^{2}}=\frac{1}{v_{p}^{2}} \phi^{(2)}-n_{p}^{(2)}+\frac{1}{2}\left[\phi^{(1)}\right]^{2}\left(\mu_{e}+\sigma_{p}^{2} \mu_{n}\right)
\end{aligned}
$$

Now using Eqs.(6) -(8), KdV equation can be readily obtain as

$$
\frac{\partial \phi^{(1)}}{\partial \tau}+A \phi^{(1)} \frac{\partial \phi^{(1)}}{\partial \xi}+B \frac{\partial^{3} \phi^{(1)}}{\partial \xi^{3}}=0
$$

where nonlinear and dissipation coefficients respectively are

$$
\begin{aligned}
& A=\frac{3}{2 v_{p}}-\frac{\left(\mu_{e}+\sigma_{p}^{2} \mu_{n}\right) v_{p}^{3}}{2} \\
& B=\frac{v_{p}^{3}}{2}
\end{aligned}
$$




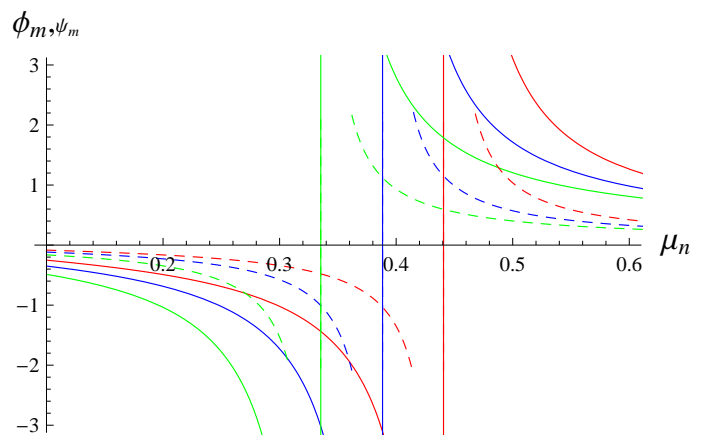

Figure 2. Variation of the amplitude of solitary (solid curve, $\phi_{m}$ ) and shock (dashed curve, $\psi_{m}$ ) waves with respect to $\mu_{n}$ for $\sigma_{p}=2, \alpha=0.7$, and $u_{0}=0.1$. Here red curve represents $\mu_{e}=0.01$, blue curve is for $\mu_{e}=0.05$, and green curve is for $\mu_{e}=0.09$

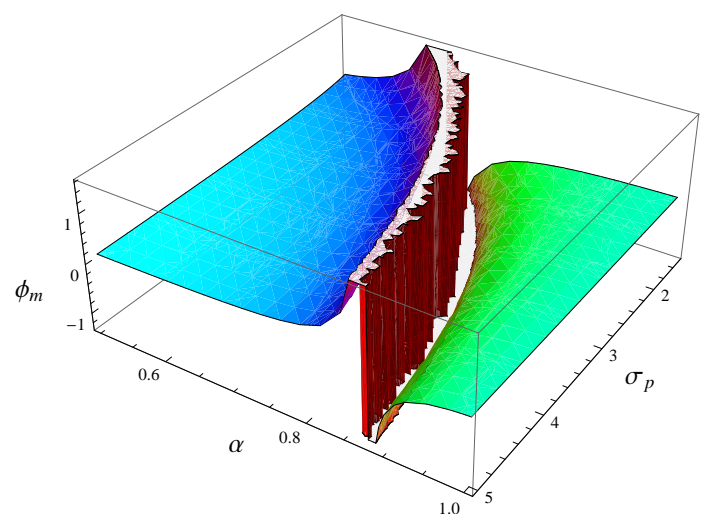

Figure 3. Variation of the amplitude of solitary wave $\left(\phi_{m}\right)$ with respect to $\alpha$ and $\sigma_{p}$ for $\mu_{e}=0.05, \mu_{n}=0.5$, and $u_{0}=0.1$.

The stationary solitary wave solution $[5,10,22,23]$ of the K-dV equation is

$$
\phi=\phi_{m} \operatorname{sech}^{2}\left[\frac{\left(\xi-u_{0} \tau\right)}{\delta_{1}}\right]
$$

where $u_{0}$ is the constant SW velocity. The amplitude $\left(\phi_{m}\right)$ and the width $(\Delta)$ of the solitary wave equation are

$$
\begin{aligned}
& \phi_{m}=\frac{3 u_{0}}{A} \\
& \delta_{1}=\sqrt{\frac{4 B}{u_{0}}}
\end{aligned}
$$

\section{Shock Wave}

To obtain Burgers equation using reductive perturbation method we have introduced the following stretched co-ordinates $[22,23]$

$$
\xi=\epsilon\left(x-v_{p} t\right)
$$




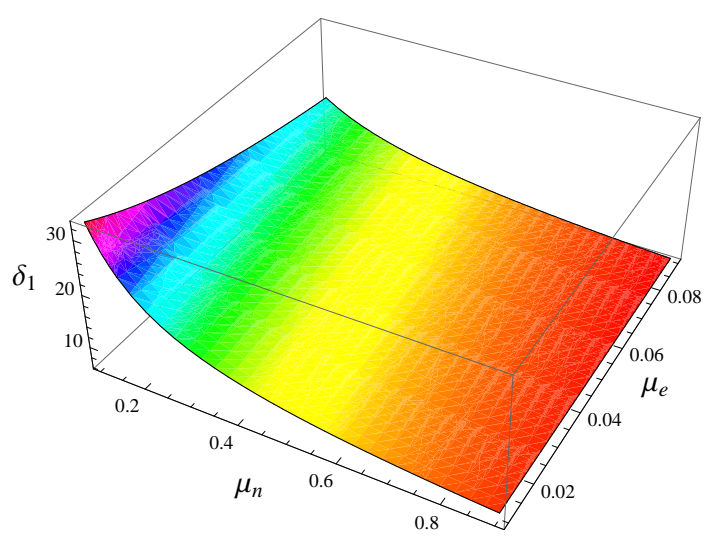

Figure 4. Variation of the width of solitary wave $\left(\delta_{1}\right)$ with respect to $\mu_{n}$ and $\mu_{e}$ for $\sigma_{p}=2, \alpha=0.7$, and $u_{0}=0.1$.

$$
\tau=\epsilon^{2} t
$$

Now, using the same procedure, done above (in Sec. 3), with the new stretched co-ordinates (for shock wave) we can obtain the linear dispersion relation similar as solitary waves, as in (5).

By following the same algebraic calculation as in Sec. 3, one can be readily obtain Burgers equation as

$$
\frac{\partial \phi^{(1)}}{\partial \tau}+A \phi^{(1)} \frac{\partial \phi^{(1)}}{\partial \xi}-C \frac{\partial^{2} \phi^{(1)}}{\partial \xi^{2}}=0
$$

where, nonlinear coefficient $A$ is the same as Eq.(10) and

$$
C=\frac{\eta}{2}
$$

The stationary solution of the Burgers equation (15) can express as $[22,23]$

$$
\psi=\psi_{m}\left[1-\tanh \frac{\left(\xi-u_{0} \tau\right)}{\delta_{2}}\right]
$$

where the amplitude and width are given by

$$
\begin{aligned}
& \psi_{m}=\frac{1}{3} \phi_{m} \\
& \delta_{2}=\frac{2 C}{u_{0}}
\end{aligned}
$$

\section{Numerical Study}

We have numerically studied the effect of non-thermal ENLIs on EPHIA solitary and shock waves in multiion plasmas with Maxwellian electrons. It is obvious from Eqs.(13), (14), (18), and (19) that $u_{0}$ is a linear function of amplitude of both solitary and shock waves and inverse function of width which means that the profile of the faster solitary and shock structure will be taller and narrower then slower one. Figure 1 shows the variation of phase speed $\left(v_{p}\right)$ with ENLIs concentration $\left(\mu_{n}\right)$ and election concentration $\left(\mu_{e}\right)$ keeping the values $u_{0}=0.1, \alpha=0.7$, and $\sigma_{p}=2$. It is found that phase velocity decreases with increasing $\mu_{n}$ and $\mu_{e}$. This is to note here that, at the critical value $(\gamma=1)$ the phase velocity $v_{p}$ reduce to $1 / \sqrt{\mu_{e}}$, which means that at critical condition, the phase velocity is independent of all the parameters except $\mu_{e}$. 


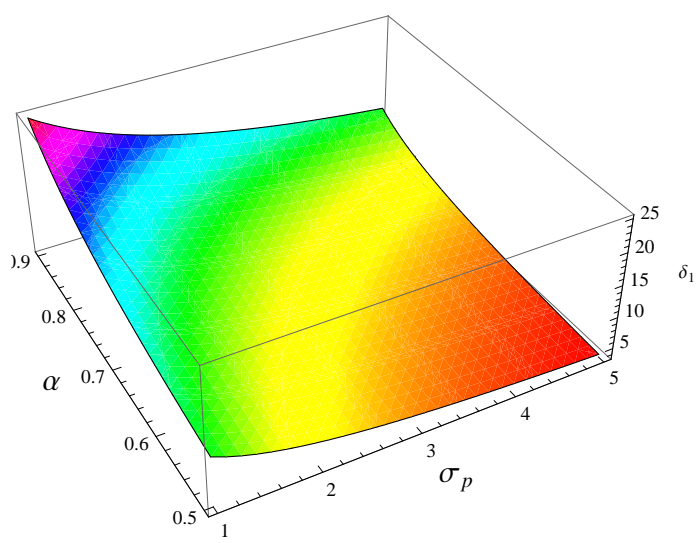

Figure 5. Variation of the width of solitary wave $\left(\delta_{2}\right)$ with respect to $\sigma_{p}$ and $\alpha$ for $\mu_{e}=0.05, \mu_{n}=0.5$, and $u_{0}=0.1$.

Depending on the value of $A$ the solitary and shock waves might be associated with positive or negative potentials. Equation (10) indicates that $A$ is a function of $\sigma_{p}, \mu_{n}, \mu_{e}$ and $\alpha$. Therefore, these parameters are responsible for the solitary waves associated with positive and negative potentials. This is also shown in figs. 2 and 3. Figure 2 shows the variation of the amplitude of solitary (solid curve, $\phi_{m}$ ) and shock (dashed curve, $\psi_{m}$ ) waves with $\mu_{n}$ keeping the values $u_{0}=0.1, \sigma_{p}=2$, and $\alpha=0.7$. The red curve corresponds to $\mu_{e}=0.01$, blue curve to $\mu_{e}=0.05$ and green curve to $\mu_{e}=0.09$. It is observed from the figure that there is a critical value of $\mu_{n}$ considering other parameters constant below (above) which the solitary and shock waves are associated with negative (positive) potentials. Similar types of criticality are also observed by Haider et al. $[8,9,10]$. It is seen from Eqs. (13) and (18) that the amplitude of the solitary wave is three times larger than that of shock waves though the critical value for solitary and shock structures is the same. This figure also represents the above statement. Above the critical value the amplitude decrease with increasing the value of $\mu_{n}$. It is also observed that increasing the value of $\mu_{e}$ the amplitude of the solitary and shock waves decreases and the critical value of $\mu_{n}$ shifted to higher value of it. Figure 3 shows the variation of the amplitude $\left(\phi_{m}\right)$ of solitary waves with $\sigma_{p}$ and $\alpha$, keeping the values $\mu_{e}=0.05, \mu_{n}=0.5$, and $u_{0}=0.1$. We have found a critical value of $\alpha$ which increases with increasing $\sigma_{p}$; below (above) which the positive (negative) solitary and shock waves are found. We have seen that amplitude $\left(\phi_{m}\right)$ increases with increasing $\alpha$ below the critical value but decreases with $\alpha$ above the critical value. Figure 4 shows the variation of the width $\left(\delta_{1}\right)$ with $\mu_{n}$ and $\mu_{e}$ keeping the values $\sigma_{p}=2, \alpha=0.7$, and $u_{0}=0.1$; which indicates that $\delta_{1}$ decreases with increasing $\mu_{n}$ and $\mu_{e}$. Figure 5 shows the variation of the width $\left(\delta_{1}\right)$ with $\sigma_{p}$ and $\alpha$ keeping the values $\mu_{e}=0.05$ and $\mu_{n}=0.5$, and $u_{0}=0.1$. We have found that $\delta_{1}$ increases with increasing $\alpha$ and decreases with increasing $\sigma_{p}$.

\section{Discussion}

EPHIA solitary and shock structures have been analyzed in an unmagnetized plasma containing EPHI fluid with nonthermal distributed ENLIs and Maxwellian electron. We have investigated the basic features of amplitude, width, and temperature effects of electrons and ions. The results that we obtained from this investigation can be summarized as follows:

1. The amplitude of the solitary waves is three times higher than shock structures.

2. The amplitude of the solitary and shock waves for nonthermal ENLIs is proportional to the wave speed $u_{0}$ whereas the width is inversely proportional to it, which means that the profile of the faster soliton will be taller and narrower than slower one.

3. Depending on the parametric regimes both the solitary and shock waves might be associated with positive or negative potentials. There must be a critical value for nonthermal ENLIs and nonthermal parameter $\alpha$ keeping other parametric regimes constant, where the amplitude goes infinity. 
4. Below (above) the critical value of ENLIs concentration the solitary and shock waves are associated with positive (negative) potentials. And also negative (positive) potentials are found below (above) the critical value of the nonthermal parameter $(\alpha)$.

5. Maxwellian electrons concentration makes the lower amplitude solitary and shock waves.

6. The width of solitary structures decreases with increasing $\alpha, \sigma_{p}, \mu_{n}$ and $\mu_{e}$; i.e. the solitary waves will be spiky for the higher values of these.

7. The width of the shock waves is a linear function of the viscus coefficient, i.e. spiky profile of the shock waves are found for the lower value of the viscus coefficient.

We hope that our present investigations may be helpful to understand the effect of heavy ion on EPHIA solitary and shock waves in multi-component electron-ion plasmas in laboratory and astrophysical system like radio wave propagation in the ionosphere [12] and also give a guideline to further development in the relevant field.

\section{References}

1. I. L. Cooney, M. T. Gavin, I. Tao, and K. E. Lonngren, "A two-dimensional soliton in a positive ion-negative ion plasma" IEEE Trans. Plasma Sci. 19, 1259 (1991).

2. Q. Z. Luo, N. D'Angelo, and R. L. Merlino, "Shock formation in a negative ion plasma" Phys. Plasmas 5, 2868 (1998).

3. S. H. Kim and R. L. Merlino, "Electron attachment to C7F14 and SF6 in a thermally ionized potassium plasma" Phys. Rev. E 76, 035401 (2007).

4. M. K. Mishra, R. S. Chhabra, and S. R. Sharma, "Obliquely propagating ion-acoustic solitons in a multicomponent magnetized plasma with negative ions" J. Plasma Phys. 52, 409 (1994).

5. F. Sayed, M. M. Haider, A. A. Mamun, P. K. Shukla, B. Eliasson, and N. Adhikary, "Dust ion-acoustic solitary waves in a dusty plasma with positive and negative ions", Phys. Plasmas. 15, 063701 (2008).

6. M. M. Haider, T. Ferdous, S.S. Duha, and A.A Mamun, "Dust-ion-acoustic Solitary Waves in Multicomponent Magnetized Plasmas", Open J. Modern Phys. 1, 13 (2014).

7. M. M. Haider, T. Ferdous, and S. S. Duha, Cent. Eur. J. Phys. 1(2), 13-24, (2014).

8. M. M. Haider, T. Ferdous, and S. S. Duha, "The effects of vortex like distributed electron in magnetized multi-ion dusty plasmas", J. Theor. Appl. Phys. 9, 159 (2015).

9. M. M. Haider, "Ion-AcousticWaves Instability in a Three Components Magneto- Plasma with Nonthermal Electrons", Contrib. Plasma Phys. 53, 234 (2013).

10. M. M. Haider, "Dust-ion-acoustic solitary structure with opposite polarity ions and non-thermal electrons", Eur. Phys. J. D. 70, 28 (2016).

11. Y. Nakamura, and I. Tsukabayashi, "Observation of Modified Korteweg-de Vries Solitons in a Multicomponent Plasma with Negative Ions" Phys. Rev. Lett. 52, 2356 (1984).

12. C. O. Hines, "Heavy-ion effects in audio-frequency radio propagation", J. Atmospheric Terrest. Phys. 11, 36 (1957).

13. M.R. Hossen, L. Nahar, S. Sultana, and A.A. Mamun, "Nonplanar ion-acoustic shock waves in degenerate plasmas with positively charged heavy ions", High Energ. Density Phys. 13, 13 (2014).

14. S. A. Ema, M. Ferdousi, S. Sultana, and A. A. Mamun, "Dust-ion-acoustic shock waves in nonextensive dusty multi-ion plasmas", Eur. Phys. J. Plus 130, 46 (2015).

15. M. G. Shah, M. M. Rahman, M. R. Hossen, and A. A. Mamun, "Roles of Superthermal Electrons and Adiabatic Heavy Ions on Heavy-Ion-Acoustic Solitary and Shock Waves in a Multi-Component Plasma", Commun. Theor. Phys. 64, 2, 208-214 (2015).

16. O. Rahman and M. M. Haider, "Modified Korteweg-de Vries (mK-dV) Equation Describing Dust-ion-acoustic Solitary Waves in an Unmagnetized Dusty Plasma with Trapped Negative Ions", Advances in Astrophysics 1, 3 (2016).

17. H. Washimi and T. Taniuti, "Propagation of Ion-Acoustic Solitary Waves of Small Amplitude", Phys. Rev. Lett. 17, 996 (1966).

18. D. J. Korteweg and G. de Vries, "On the change of form of long waves advancing in a rectangular canal, and on a new type of long stationary waves", Philosophical Magazine 39, 422 (1895).

19. J. M. Burgers, "A mathematical model illustrating the theory of turbulence", In Advances in applied mechanics 1, 171, (1948). 
20. R. A. Cairns, A. A. Mamun, R. Bingham, and R. Boström, R. O. Dendy, C. M. C. Nairn, and P. K. Shukla, "Electrostatic solitary structures in non-thermal plasmas", Geophys. Res. Lett. 22, 2709 (1995).

21. E. Witt and W. Lotko, "Ion-acoustic solitary waves in a magnetized plasma with arbitrary electron equation of state", Phys. Fluids, 26, 2176 (1983).

22. M. M. Haider, "Soliton and Shock Profiles in Electron-positronion Degenerate Plasmas for Both Nonrelativistic and Ultra-Relativistic Limits", Z. Naturforsch A 71 (12), 1131 (2016).

23. M. M. Haider and A. Nahar, "Dust-Ion-Acoustic Solitary and Shock Structures in Multi-Ion Plasmas with Super-Thermal Electrons", Z. Naturforsch A 72 (7), 627 (2017). 